\title{
PARAMETERS OPTIMIZATION FOR A NOVEL VACUUM LASER ACCELERATION TEST AT BNL-ATF*
}

\author{
L. Shao ${ }^{\#}$, D. Cline, Feng Zhou \\ University of California at Los Angeles, CA 90095, U.S.A. \\ ATF, Brookhaven National Lab, Upton, NY 11973, U.S.A.
}

\begin{abstract}
This paper presents a new VLA (Vacuum Laser Accelerator) theory model which has revealed that the injection electrons with low energy and small incident angle relative to the laser beam are captured and significantly accelerated in a strong laser field. For the further step for verifying the novel-VLA mechanics, we propose to use the BNL-ATF Terawatt $\mathrm{CO}_{2}$ laser and a high-brightness electron beam to carry out a proof-ofprinciple beam experiment. Experiment setup including the laser injection optics and electron extraction system and beam diagnostics is presented. Extensive optimized simulation results with ATF practical parameters are also presented, which shows that even when the laser intensity is not very high, the net energy gain still can be seen obviously. This could be prospect for a new revolution of vacuum laser acceleration.
\end{abstract}

\section{THEORETIC BACKGROUD}

As we all known for long time, the problem of laserelectron acceleration in vacuum is the phase velocity of the laser field. The famous Lowson-woodward theorem $[1,2]$ states that a relativistic electron cannot gain net energy by interacting with a laser field in vacuum. The key points are the phase velocity slippage experienced by electron is so fast that electron cannot get net energy after interacting with the laser beam and the interaction region is infinite extent. However, recently this theory is circumvented by both experiment [3] and simulation [4$6]$. In the cases of very intense laser beam we have found a very special important feature in the focused laser beam [7]. That is in the focused field in vacuum, there exists a apecial region characterized by subluminous wave phase velocity. Due to this feature we are able to propose a whole new acceleration scheme. In the subluminous phase velocity region the electron has possibility to match the acceleration part of the phase and can be captured in it gaining net energy exchange by being accelerated. The phase velocity is described as the equation,

$$
\partial \varphi / \partial t+\left(v_{\varphi}\right)_{J} \cdot(\nabla \varphi)_{J}=0
$$

$(\nabla \varphi)_{J}$ is the effective phase velocity of the wave along a particle trajectory. The effective phase velocity experienced by the electron also depends on the intensity of the laser beam $a_{0}=e E_{0} / m \omega c$, which is a dimensionless parameter measuring the intensity, and the some other parameters of incident conditions, such as initial electron momentum, incident angle and initial phase of the laser

*Work supported by DOE Grant

"leishao@physics.ucla.edu beam. These parameters need to adjust simultaneously to get an optimal condition for the acceleration.

\section{EXPERIMENT CONDITION AT ATF}

The current ATF $\mathrm{CO}_{2}$ Laser can operate at $25 \mathrm{ps}$ pulse length (FWHM) with 10J energy. In the near future the ATF $\mathrm{CO}_{2}$ laser can operate at the pulse length reducing to $2 \mathrm{ps}$, under which condition if we can focus the power $5 \mathrm{TW}$ into an area with radius of $20 \mu \mathrm{m}$, then we can have $a_{0}$ reach 5.7 which is strong enough for laser-electron acceleration in our simulation. And the relation of laser beam waist $w_{0}$ with $f / D$ of a parabolic mirror is:

$$
2 w_{0}=\frac{4 \lambda}{\pi} \cdot \frac{f}{D}
$$

Where $\lambda=10.6 \mu \mathrm{m}$ is the laser wave length, $f$ is the focal length and $D$ is the input $\mathrm{CO}_{2}$ laser beam diameter. The interaction device can be designed like fig. 1 . The solid lines and the dot lines show that the laser direction can be adjusted by rotating the parabolic mirror with specified angle. In order to get $r=20 \mu \mathrm{m}$, we shall use $\mathrm{f} / \mathrm{D}$ 3. Such a short-focal-length copper parabolic mirror is available from commercial companies.

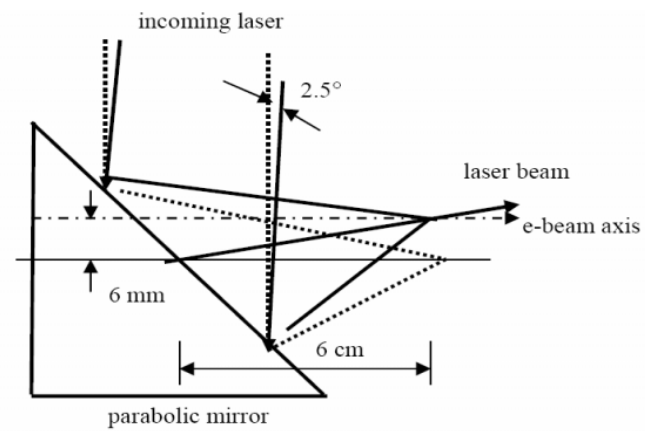

Figure 1: Schematic layout of the laser beam injection to the electron beam; dimensions in the plot are not scaled.

As our design, the laser would focus and continue propagating after reflecting on the parabolic mirror. And the electron beam can go through the hole made on the parabolic mirror and meet the laser and begin to interact in the propagation direction. The initial incident angle can be adjusted by the parabolic mirror. Normally the incoming laser (dot-line in the figure) is injected and focused by a $45^{\circ}$ parabolic copper mirror with $6 \mathrm{~cm}$ focal length, and the focused laser beam (dot-line) is collinear 

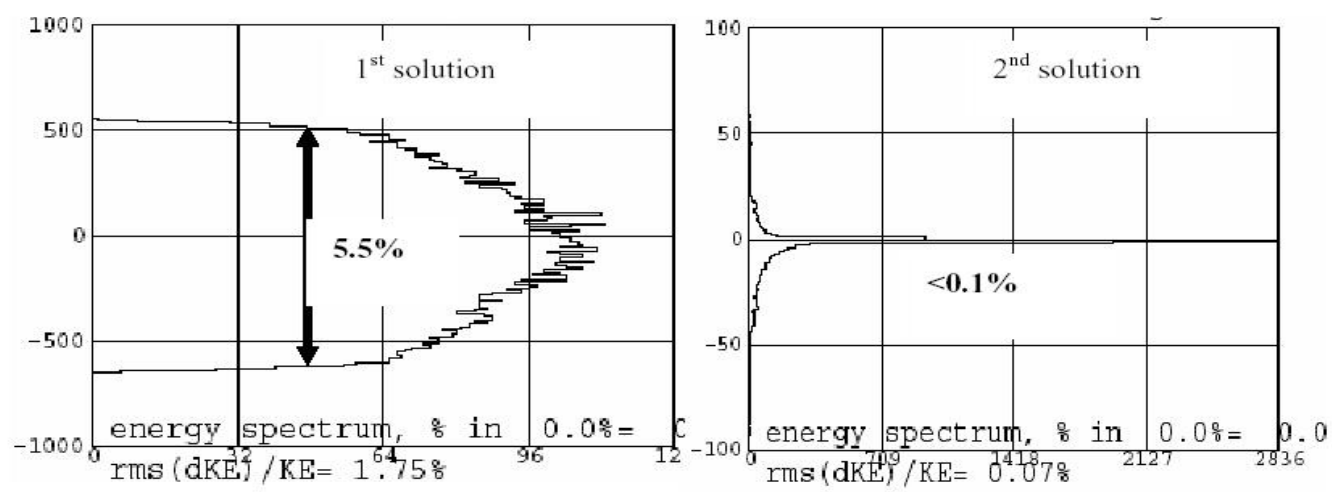

Figure 2: PARMELA simulations for beam energy spectrum for the two solutions at 20MeV; ordinate axis is particle number; abscissa axis is the energy offset from the central energy in units of keV.

with e-beam, which is basically parallel to the parabolic axis, as shown in figure 1 . However, when the incoming laser (solid line) has a small rotation angle $\left(2.5^{0}\right)$ to the dot-line by manipulating the input laser optics, the laser beam has a $5^{0}$ incident angle to the e-beam. The e-beam axis needs to be shifted from the parabolic mirror central axis by $6 \mathrm{~mm}$ if the focal length of the mirror is $6 \mathrm{~cm}$. a small hole needs to be drilled for the -beam transport.

As for the electron beam, ATF operates at high energy $(\geq 40 \mathrm{MeV})$ routinely. However, this VLA test needs a low-energy beam $(\leq 20 \mathrm{MeV})$. The ATF has two SLACtype S-band linac sections. Basically there are two solutions to obtain a lower-energy beam: one is to adjust the first linac section phasing in acceleration with a larger accelerating gradient but phasing the second linac section in deceleration to obtain a lower energy; and the other is to adjust both linac sections phasing in acceleration but with a lower accelerating gradient. The electron beam energy spectrum was simulated by PARMELA code. As shown in Fig. 2, the second solution can offer a smaller energy spread. Therefore, the second solution was used to tune the real beam, and it was successfully tuned to the eng of the beam line at $20 \mathrm{MeV}$ beam energy. The achieved beam test results at $200 \mathrm{pC}$ are summarized: normalized transverse emittance is below $3.5 \mu \mathrm{m}$, energy spread smaller than $0.15 \%$. The next step is to tune $15 \mathrm{MeV}$ or even lower energy beam to the end of beam line, which allows us to choose the e-beam energy

\section{SIMULATION RESULTS AND ANALYSIS}

We have made lots of simulation work under several sets of different parameter in order to find the range of optimal parameters for the acceleration. Here we just choose an example to present such a new acceleration scheme with the important feature of subluminous phase velocity.

Figure 3 shows the typical case of CAS. Figure 3(a) present the electron energy as a function of time. From which we can see that the electron gets net energy obviously after the interaction region. Therefore we can export the electron with high net energy. Figure 3(b)
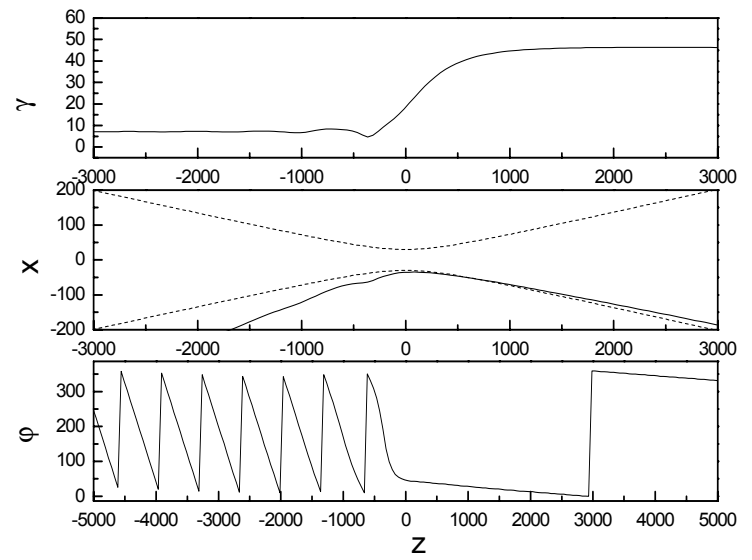

Figure 3: One typical case of electron dynamics. A continuous laser beam with field intensity $a_{0}=5$ is used.

Other parameters are $k w_{0}=30$ of the laser beam waist, $P_{x i}=0.7, P_{y i}=0$ and $P_{z i}=7$, the initial momentum of the electron. (a) Electron energy $\gamma$ as a function of time. (b) Electron trajectory in the $x-z$ plane. (c) The laser wave phase experienced by the electron as a function of time.

shows the trajectory of the electron. It clearly indicates that the electron goes with staying in the laser beam, especially along the outline of the laser beam, where is the region with sublumious phase velocity. Figure 3(c) shows the variation of laser phase experienced by an electron during interacting. The most meaningful phenomenon shown by it is that after electron passing the waist of laser beam, which is the major part of the interaction, the phase slippage between the electron and the laser wave becomes low obviously. That means there is possibility for the electron keep staying in the acceleration phase and get net energy exchange from the laser beam finally. All of this is due to the special feature of the focused laser beam. There are some regions in 
which the phase velocity is apparently lower than $c$, the light speed in vacuum.

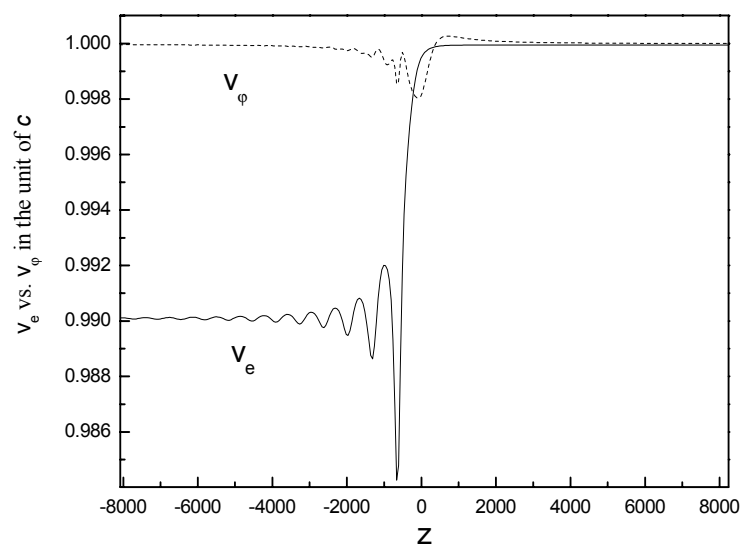

Figure 4: The solid-line and dot-line demonstrate the variations of electron's velocity and phase velocity along the electron trajectory respectively. Other parameters are as the same as Figure 3.

Figure 4 shows the electron's velocity and phase velocity along the electron trajectory during the whole interacting process. We can see that at the region close to the laser waist the phase velocity becomes very low, which is even lower than the meanwhile electron's velocity, while the electron has vibrated to a high energy. Therefore, the electron can catch up with the part of acceleration phase and be captured and accelerated by the laser continuously.

After the simulation on different parameters we found that the initial energy of electrons is a very important parameter. The initial electron energy should not be very high to prevent from penetrating the beam quickly. The longitudinal direction is the main direction to accelerate the electron. As shown in Figure 5, with the initial energy increasing the net energy gain decreases sharply. Also the initial energy chosen here is according to the current experiment condition in ATF. Theoretically, the lower initial energy should be the better.

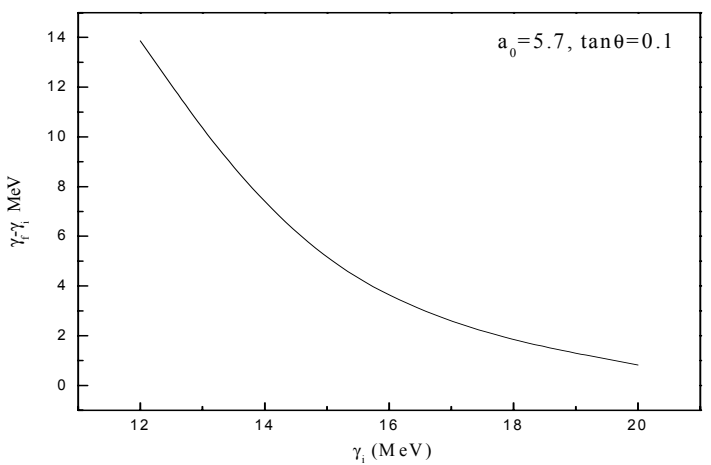

Figure 5: The gain net energy of an electron versus the initial incident energy of the electron. Here we use the laser beam with intensity of $a_{0}=5.7$, the laser beam waist $k w_{0}=24$ and incident angle $\tan \theta=0.1$.

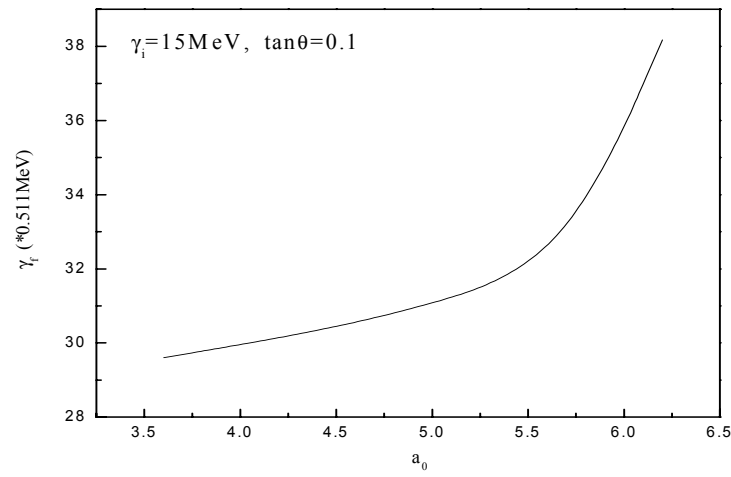

Figure 6: The gain net energy of an electron versus the intensity of laser field. The initial energy of electron is $15 \mathrm{MeV}$; other parameters are the same as Figure 5.

As for the laser intensity, the higher intensity gives out the better result. So far, we know that the threshold value of laser intensity in this scheme is near $a_{0} \approx 5$ it's shown in Figure 6. Therefore, we should choose the electron with the energy as low as we can.

\section{CONCLUSION}

- An electron can get net energy exchange from the focused laser beam in vacuum due to the special feature of subluminous phase region.

- In order to get better result we need to adjust the parameters get optimal choice.

- And of course the intensity of laser beam is the critical parameter. High intensity laser field is expected to get more optimal result.

\section{REFERENCES}

[1] P.M. Woodward, J. IEE 93, $1554 \sim 1947$ !; J.D. Lawson, IEEE Trans. Nucl. Sci. 26, 4217 1979!; R.B. Palmer, Part. Accel. 11, 81 (1980).

[2] E. Esarey, P. Sprangle, and J. Krall, Phys. Rev. E 52, 5443 (1995); P. Sprangle, E. Esarey, and J. Krall, Phys. Plasmas 3, 2183 (1996).

[3] G. Malka, E. Lefebvre, J.L. Miquel, Phys. Rev. Lett. 78 (1997) 3314; G. Malka, E. Lefebvre, J.L. Miquel, Phys. Rev. Lett. 80 (1998) 1352.

[4] B. Quesnel, and P. Mora, Phys. Rev. E 58, 3719 (1998).

[5] F.V. Hartemann et al., Phys. Rev. E 58, 5001 (1998).

[6] G.V. Stupakov and M.S. Zolotorev, Phys. Rev. Lett. 86, 5274 (2001).

[7] J. Pang et. al., Phys. Rev. E 66. 0565 (2002) 